\title{
Effects of initial coherence on distinguishability of pure/mixed states and chiral stability in an open chiral system
}

\author{
Heekyung $\operatorname{Han}^{1, *}$ and David M. Wardlaw ${ }^{1,2, \dagger}$ \\ ${ }^{1}$ Department of Chemistry, Queens University, Kingston, Ontario KYL , Canada \\ ${ }^{2}$ Department of Chemistry, University of Western Ontario, \\ London, Ontario N6H 5B\%, Canada
}

(Dated: November 4, 2018)

\begin{abstract}
We examine how initial coherences in open chiral systems affect distinguishability of pure versus mixed states and purity decay. Interaction between a system and an environment is modeled by a continuous position measurement and a two-level approximation is taken for the system. The resultant analytical solution is explored for various parameters, with emphasis on the interplay of initial coherences of the system and dephasing rate in determining the purity decay and differences in the time evolution of pure vs. mixed initial states. process. Implications of the results on several fundamental problems are noted.
\end{abstract}

${ }^{*} \mathrm{E}$-mail address: hhan0410@gmail.com

${ }^{\dagger} \mathrm{E}$-mail address: dwardlaw@uwo.ca 


\section{INTRODUCTION}

The quantum principle of parity conservation prohibits the presence of the left- and righthanded states of chiral molecules as true stationary states, since the Hamiltonian and the parity operator commute and thus the eigenfunctions of the parity-invariant Hamiltonian should show parity symmetry in the absence of energy degeneracy [1]. However, some chiral molecules are observed to be stable for an enormously long time despite their lack of parity symmetry. Theoretical efforts to resolve this conundrum date back to Hund's proposal in the early days of quantum-mechanics [2]. He formulated a description of the stability of chiral molecules in terms of quantum tunneling in a symmetrical double-well potential, where exceedingly long tunneling times through the inter-well potential barrier were responsible for the stability. However, one can show that the tunneling time estimates in observed stable chiral molecules have a wide range, from sub-picosecond to the age of the universe. On the other hand, there have been attempts to employ the interaction of molecules with the environment, which leads to decoherence, and thus to a suppression of coherent tunneling oscillations, to explain the stability of chiral molecules. Intermolecular collisions [3], interaction with photons [4], and with phonons [5] have been introduced as the physical origin of the dephasing process.

Another aspect of stabilization of chiral molecules is the experimental realization of the superpositions of stable chiral states (so-called Schrödinger's cat state). Several proposals have been suggested for the preparation and detection of the superposed chiral states utilizing phase-controlled ultrashort laser pulses [6, 7], and growing attention has been directed towards using lasers to manipulate the molecular chirality [8, 9]. Approaches such as these which rely on the interaction between an external electric field and a molecule have become more promising with recent advances in laser technology. Considering that the decoherence effect is crucial to experimental realizations of coherent superposition of chiral states, it is thus of great interest to understand the coherent tunneling dynamics of chiral systems coupled to external environments more thoroughly.

In this Letter, we study the role of initial coherences, in an open chiral system, on the distinguishability of initially pure versus initially mixed states as measured by population difference between the chiral states, and the vulnerability to decoherence as measured by the purity decay. The influence of an environment on the system is modeled by continuous 
position measurements, an approach often used in the studies of the quantum Zeno effect [10] and in the studies of decoherence effects in chemical reactions [11]. In particular,

Milburn and his coworkers $[12,13]$ studied a model of continuous position measurements on a quantum system in a double-well potential, and within a two-level approximation their resultant master equation is identical to ours. However, their goal was the demonstration of the quantum Zeno effect in certain parameter regimes and thus an analytical solution was obtained only for an initially localized state. In contrast, our goal is the identification of the roles of the initial coherences and the subsequent decoherence in the distinguishability of pure vs. mixed chiral states and the stabilization (or loss) of the chirality. Accordingly we have obtained an analytical solution for an arbitrary initial condition and explored this solution for a variety of initial conditions and dephasing rates. Our results provide some answers to three fundamental questions arising in open chiral systems: 1) Can we distinguish a pure state from a mixture with same populations as those of the pure state? 2) Why are some chiral systems stable for a very long time? 3) Why are superpositions of stable chiral states not observed experimentally? Distinguishability between the initially pure state and the initially mixed state is measured by a population difference between the two chiral states. The stability of the system is measured by the purity, which is equal to 1 only for a pure state and decays as coherences are lost during an interaction between the system and the environment.

\section{CHIRAL SYSTEMS IN THE PRESENCE OF DEPHASING}

\section{A. Formalism (a two-level approximation)}

Consider chiral molecules in a symmetric double-well potential interacting with a bath and assume that the effects of collisions between the system and the environment can be modeled by the continuous measurement of the system coordinate, say $\hat{x}[14,15]$. Then the system density operator $\hat{\rho}$, which is obtained by tracing the total density operator over the environment, obeys the master equation [11],

$$
\frac{\partial \hat{\rho}}{\partial t}=-\frac{i}{\hbar}\left[\hat{H}_{0}, \hat{\rho}\right]-\Gamma[\hat{x},[\hat{x}, \hat{\rho}]] .
$$


Here $\hat{H}_{0}$ is the system Hamiltonian and $\Gamma$ is a coupling strength between the system and the bath. The second term in this equation, a multiplication of $\Gamma$ and the double commutator, represents the environmental effect on the system, and is expected to destroy coherences between the eigenstates of the position measurement operator, $\hat{x}$. This loss of coherence, i.e., decoherence, has been argued as the essential ingredient to bridging the gap between classical and quantum descriptions of a microscopic system coupled to its environment [16].

To invoke a two-level approximation, we assume that for an isolated system the potential barrier is very high or equivalently the energy of the system is sufficiently low that the higher energy levels are not involved in the dynamics. In addition, since this is an open system, we should point out that the position measurement of the system is known to cause its energy and its energy width to increase over time $[11,17]$. The system energy and energy width will eventually become greater than the potential barrier, leading to the breakdown in the validity of the two-level approximation. The stronger $\Gamma$, the shorter the time during which the two-level approximation will be valid.

Within the two-level approximation, we need only the two lowest energy eigenstates, $|+\rangle$ (even parity; ground state) and $|-\rangle$ (odd parity) with energies $E_{+}$and $E_{-}$. Alternatively, we can consider the two localized states $|L\rangle$ and $|R\rangle$, found in the left- and right-hand wells, respectively. By convention, these states are given by $|L\rangle=\frac{1}{\sqrt{2}}(|+\rangle+|-\rangle)$ and $|R\rangle=$ $\frac{1}{\sqrt{2}}(|+\rangle-|-\rangle)$. The free Hamiltonian readily takes the form $\hat{H}_{0}=E_{+}|+\rangle\left\langle+\left|+E_{-}\right|-\right\rangle\langle-|=$ $E_{m}(|R\rangle\langle R|+| L\rangle\langle L|)-\delta(|L\rangle\langle R|+| R\rangle\langle L|)$ where $E_{m}=\frac{\left(E_{+}+E_{-}\right)}{2}$ and $\delta=\frac{\left(E_{-}-E_{+}\right)}{2} \equiv \hbar \omega$ with a tunneling frequency $\omega$. For simplicity, choosing the energy origin to correspond to $E_{m}=0$, i.e.,$E_{+}=-E_{-}$and using the usual Pauli matrices $\hat{\sigma_{x}}, \hat{\sigma_{y}}, \hat{\sigma_{z}}$ in the $|L\rangle$ $|R\rangle$ basis, $H_{0}$ becomes $H_{0}=-\delta \hat{\sigma_{x}}$. Using the two-level approximation and the evenness and oddness of $|+\rangle$ and $|-\rangle$, respectively, we derive the position measurement operator as $\hat{x}=|+\rangle\langle+|\hat{x}|-\rangle\langle-|+$ H.c $=x_{\mathrm{avg}} \hat{\sigma}_{z} \approx x_{\min } \hat{\sigma}_{z}$, where H.c denotes the Hermitian conjugate and $\pm x_{\min }$ are the positions of the double-well minima, which for a deep well are very close to the average positions of the right and left wave functions (denoted by $\pm x_{\text {avg }}$ ). Finally the master equation for the two-level system becomes [12]

$$
\frac{\partial \hat{\rho}}{\partial t}=\frac{i}{\hbar}\left[\delta \hat{\sigma}_{x}, \hat{\rho}\right]-\gamma\left[\hat{\sigma}_{z},\left[\hat{\sigma}_{z}, \hat{\rho}\right]\right]
$$

with $\gamma \equiv \Gamma x_{\min }^{2}$. This equation gives the evolution of the tunneling two-level system sub- 
jected to a continual measurement of the system coordinate $\hat{x}$ as represented by the Pauli matrix $\hat{\sigma}_{z}$. The second term in this equation represents a measurement of a population difference between two states $|L\rangle$ and $|R\rangle$, and leads to decay of the coherences between the $|L\rangle$ and $|R\rangle$ states, i.e. , of the off-diagonal matrix elements, $\rho_{L R}$. Note that here $\gamma$ is given by "a multiplication of $\Gamma$ by $x_{\min }^{2}$ ", which means that the magnitude of $\gamma$ is determined by the interaction strength (between the system and the bath) and the system potential as well. Thus within the two-level approximation, $\gamma$ can be increased by increasing the interaction strength or by increasing the distance between two wells of the potential. It is intuitive that for a system composed of two wavepackets that are initially spatially separated and subjected to an environment destroying coherences between these two wavepackets, the larger the distance between two wavepackets and the larger the interaction strength between the system and the environment, the faster coherences between two wavepackets are lost [10].

It is also noteworthy that this master equation is identical to that resulting from a twolevel atom driven by a field undergoing a pure dephasing [18] in quantum optics, as previously mentioned in Ref. $[12,13]$. This equivalency emerges where the following replacements are made: $|L, R\rangle \rightarrow$ the two energy eigenstates, $\omega \rightarrow$ Rabi-frequency, and $\gamma \rightarrow$ pure dephasing rate.

The density-matrix elements of the states $|L\rangle$ and $|R\rangle, \rho_{L L}, \rho_{R R}$, and $\rho_{L R}$ obey the following set of equations:

$$
\begin{aligned}
& \frac{\partial \rho_{L L}}{\partial t}=-\operatorname{Im}\left(2 \omega \rho_{R L}\right), \\
& \frac{\partial \rho_{R R}}{\partial t}=\operatorname{Im}\left(2 \omega \rho_{R L}\right), \\
& \frac{\partial \rho_{R L}}{\partial t}=-4 \gamma \rho_{R L}+i \omega\left(\rho_{L L}-\rho_{R R}\right)
\end{aligned}
$$

It is convenient to switch to the Bloch-vector representation by defining the real quantities, $X, Y$, and $Z$ as $X=2 \operatorname{Re}\left(\rho_{L R}\right), Y=2 \operatorname{Im}\left(\rho_{L R}\right)$, and $Z=\rho_{R R}-\rho_{L L}$ [19]. (In regard to relationship of $X, Y$, and $Z$ to coherence and localization of the system, it is noteworthy that for a pure state, since $X^{2}+Y^{2}+Z^{2}=1$, the localized state, say $|R\rangle$, corresponds to $Z^{2}=1$, i.e., $X^{2}+Y^{2}=0$, and the strongly delocalized state corresponds to $Z^{2} \approx 0$, i.e. , 
$X^{2}+Y^{2} \approx 1$.) Rewriting Eqs. (3) to (5) in terms of $X, Y$, and $Z$ :

$$
\begin{aligned}
& \frac{d X}{d t}=-4 \gamma X \\
& \frac{d Y}{d t}=-4 \gamma Y+2 \omega Z \\
& \frac{d Z}{d t}=-2 \omega Y .
\end{aligned}
$$

The resultant equations are of standard form [20]. Thus the study of chiral stability is reduced to the solution of the $3 \times 3$ dynamical system which depend upon two parameters $\omega$ and $\gamma$. Note that the longtime steady-state solution of Eqs.(6)-(8) is $X=Y=Z=0$ as $t \rightarrow \infty$. It can be obtained by setting $d X / d t=d Y / d t=d Z / d t=0$. This stationary state, which is reached regardless of initial conditions, system parameters, or dephasing rate, represents a fully mixed state, with equal populations in the right and left wells and with no remaining coherence between them.

With an arbitrary initial state $\left[X=X_{0}, Y=Y_{0}, Z=Z_{0}\right]$, the general solution is, for $\omega>\gamma$ (tunneling dominant region),

$$
\begin{aligned}
& X=X_{0} e^{-4 \gamma t} \\
& Y=-\frac{e^{-2 \gamma t}}{2 \omega}\left[\omega Y_{0}\left\{-2 \cos (s t)+\frac{4 \gamma}{s} \sin (s t)\right\}-Z_{0}\left(s+\frac{4 \gamma^{2}}{s}\right) \sin (s t)\right], \\
& Z=e^{-2 \gamma t}\left[Z_{0}\left\{\cos (s t)+\frac{2 \gamma}{s} \sin (s t)\right\}-\frac{2 \omega Y_{0}}{s} \sin (s t)\right],
\end{aligned}
$$

with $s=2 \sqrt{\omega^{2}-\gamma^{2}}$. The population of the system in either well $\left(\rho_{R R}=\frac{1+Z}{2}, \rho_{L L}=\frac{1-Z}{2}\right)$ oscillates at the modified frequency $s$, that is determined by relative size of the dephasing rate $\gamma$ and the so-called tunneling frequency $\omega$, under an exponentially decaying envelope. The introduction of decoherence increases the period of the oscillation (since the frequency $s$ decreases as $\gamma$ increases from zero towards $\omega$ ) while simultaneously suppressing the amplitude via the decay factor $e^{-\gamma t / 2}$ in the tunneling dominant region.

On the other hand, for $\gamma>\omega$ (dephasing dominant region), the general solution is obtained by replacing $\sin (s t)$ by $\sinh (\tilde{s} t)$ and $\cos (s t)$ by $\cosh (\tilde{s} t)$ with $\tilde{s}=2 \sqrt{\gamma^{2}-\omega^{2}}$ in Eqs. (9)-(11):

$$
X=X_{0} e^{-4 \gamma t}
$$




$$
\begin{aligned}
Y & =-\frac{e^{-2 \gamma t}}{2 \omega}\left[\omega Y_{0}\left\{-2 \cosh (\tilde{s} t)+\frac{4 \gamma}{\tilde{s}} \sinh (\tilde{s} t)\right\}-Z_{0}\left(\tilde{s}+\frac{4 \gamma^{2}}{\tilde{s}}\right) \sinh (\tilde{s} t)\right] \\
Z & =e^{-2 \gamma t}\left[Z_{0}\left\{\cosh (\tilde{s} t)+\frac{2 \gamma}{\tilde{s}} \sinh (\tilde{s} t)\right\}-\frac{2 \omega Y_{0}}{\tilde{s}} \sinh (\tilde{s} t)\right]
\end{aligned}
$$

In this regime for an initially localized state, say $|R\rangle$, the system approaches the longtime steady-state monotonically without any oscillations present. Ref.[12] demonstrated the quantum Zeno effect in this regime: when the measurement strength exceeds some threshold, the system initially prepared in one well remains stuck on a time scale of the measurement strength. If we attempt to apply this observation to our open chiral system, then we may say that for an initially chiral system the time over which the chirality is preserved will increase proportionally when $\gamma$ increases beyond a certain threshold (here $\omega$ ). Indeed, this is what we observe in the case with an initially localized state for a dephasing dominant region in Sec.II C. Further, considering that previous studies focused on manifestation of the quantum Zeno effect for initially localized states, it would be interesting to explore whether and how this quantum Zeno effect, namely, the suppression of a transition (tunneling), exists in the case of an initially delocalized state; this will be reported in Sec.II C.

\section{B. Distinguishability}

In this subsection, we examine how the initial coherences affect, over time and under the influence of decoherence, the distinguishability between the initially pure state and the mixed state. These comparisons require selection of initial conditions for the pure state $\left(X_{0}^{\mathrm{P}}, Y_{0}^{\mathrm{P}}, Z_{0}^{\mathrm{P}}\right)$ and the mixed state $\left(X_{0}^{\mathrm{M}}, Y_{0}^{\mathrm{M}}, Z_{0}^{\mathrm{M}}\right)$. The most meaningful comparison arises from assigning the same initial population of $|L\rangle$ and $|R\rangle$ in the initial $(t=0)$ pure and mixed states, i.e. $Z_{0}^{\mathrm{P}}=Z_{0}^{\mathrm{M}}$, but choosing the degree of initial coherence to be zero for the

mixed state (maximum randomness scenario), i.e. $X_{0}^{\mathrm{M}}=Y_{0}^{\mathrm{M}}=0$, while varying $Y_{0}^{\mathrm{P}}$ for the pure state. Note that $X_{0}^{\mathrm{P}}$ is determined by $\left\{X_{0}^{\mathrm{P}}\right\}^{2}+\left\{Y_{0}^{\mathrm{P}}\right\}^{2}+\left\{Z_{0}^{\mathrm{P}}\right\}^{2}=1$, and $X_{0}^{\mathrm{P}}$ will not affect the distinguishability as measured by $Z$ since $X$ and $Z$ are decoupled (see Eqs. (6)(8)). As easily can be seen from Eqs.(11) and (14), the difference between the time-evolution of $Z$ of the initially pure state and that of the corresponding mixed state, $\Delta Z\left(\equiv Z^{\mathrm{P}}-Z^{\mathrm{M}}\right)$ 
is given by:

$$
\begin{aligned}
& \Delta Z(t)=-\frac{2 \omega Y_{0}^{\mathrm{P}}}{s} e^{-2 \gamma t} \sin (s t) \text { for } \omega>\gamma, \\
& \Delta Z(t)=-\frac{\omega Y_{0}^{\mathrm{P}}}{\tilde{s}}\left[e^{(\tilde{s}-2 \gamma) t}-e^{-(\tilde{s}+2 \gamma) t}\right] \text { for } \omega<\gamma .
\end{aligned}
$$

From the above results one can discuss the distinguishability between the initially pure state and the corresponding initially random mixture by measuring the population difference $Z$ in the presence/absence of the bath effect destroying the right-left coherences. Note that the distinguishability is not affected by $X_{0}^{\mathrm{P}}$ as expected and that it depends strongly on the magnitude of $Y_{0}^{\mathrm{P}}$. Clearly, if $Y_{0}^{\mathrm{P}}=0, \Delta Z(t)=0$ for all time and for all $\gamma$. That is, if $Y_{0}^{\mathrm{P}}=0$, one can not distinguish the initially pure state from the corresponding randommixed state by measuring $Z$, regardless of the decoherence effect. However, if $Y_{0}^{\mathrm{P}} \neq 0$ the distinguishability does exist, although the decoherence effect tends to extinguish it over time because the decoherence forces both the initially pure state and the corresponding mixture to the maximally randomized stationary state (equal $|L\rangle$ and $|R\rangle$ populations without any remaining coherences between the two states at long times). It is noteworthy that our result reproduces, in the limit of no bath and no time-evolution of the system, the work by Harris and his coworkers [21]. They assumed essentially instantaneous measurements, occurring on a time scale short compared to that for tunneling and dephasing, and, in the framework of the wavefunction, showed that no parity sensitive experiment can measure the difference between them if the states $|L\rangle$ and $|R\rangle$ are taken to be real for the pure state, which corresponds to the case of $Y_{0}^{\mathrm{P}}=0$.

The behavior of the distinguishability also depends on the relative size of the dephasing rate and the tunneling frequency. For the tunneling dominant region $(\omega>\gamma), \Delta Z(t)$ shows oscillations that are exponentially damped with time, and whose amplitude at a given time tends to increase with increasing $\omega$ and $Y_{0}^{\mathrm{P}}$. On the other hand, for the dephasing dominant region $(\omega<\gamma),|\Delta Z(t)|$ shows a simpler behavior: it increases up to maximum at $t=t_{\max }$ from zero at $t=0$, and then decreases to zero as time increases. Here $t=t_{\max }=\frac{1}{2 \tilde{s}} \ln \left[\frac{\tilde{s}+2 \gamma}{-\tilde{s}+2 \gamma}\right]$ is obtained such that $\left.\frac{d(\Delta Z(t))}{d t}\right|_{t=t_{\max }}=0$.

Typical behavior of $\Delta Z(t)$ for several values of $\gamma$ for a given value of $\omega$ (here chosen as 1 ) is shown in Fig. 1. For $\omega>\gamma$ (tunneling dominant region, here $\gamma<1$ ), as $\gamma$ increases, the period of the oscillation increases and the amplitude decays faster, leading $\Delta Z$ to a faster 
approach toward zero $(\Delta Z=0$ means no distinguishability). For example, for $\gamma=0.5$ [upper panel], upon the measurement of population after $t=4$, one can not tell whether the system was initially in the pure state or in the corresponding mixture. On the other hand, for $\omega<\gamma$ (dephasing dominant region, here $\gamma>1$ ), after hitting the minimum point, $\Delta Z / Y_{0}^{\mathrm{P}}$ just increases monotonically towards 0 . It is noteworthy that, as $\gamma$ increases, the distinguishability disappears more slowly, while the maximum of $\left|\Delta Z / Y_{0}^{\mathrm{P}}\right|$ gets smaller and is obtained at earlier time, i.e. , $t_{\max }$ gets smaller. For instance, compare the case $\gamma=1.5$ and $\gamma=10$ in the lower panel. For the case $\gamma=1.5,\left|\Delta Z / Y_{0}^{\mathrm{P}}\right|=0.275$ at $t=t_{\max }=0.42$, and almost zero at $t=8$, indicating no distinguishability for $t>8$, while, for the case $\gamma=10,\left|\Delta Z / Y_{0}^{\mathrm{P}}\right|=0.0494$ at $t=t_{\max }=0.14$, and still nonzero as 0.0226 (roughly half of maximum $\left.\left|\Delta Z / Y_{0}^{\mathrm{P}}\right|\right)$ at $t=8$, indicating the long-time surviving distinguishability. This observation suggests that considerably strong dephasing may unexpectedly afford means to observe the long-time surviving distinguishability.

\section{Decoherence (purity decay)}

In this subsection we examine how initial coherences affect the decoherence process. In order to measure coherence decay, i.e. , decoherence, we use the purity, $\varsigma$ defined as [22] $\varsigma \equiv \operatorname{Tr}\left(\hat{\rho}^{2}\right)$, where Tr denotes a trace over the system of interest. Note that the decoherence process does not preserve the purity of the state, that is, the purity of the resultant mixed state becomes less than 1, while that of the pure state is 1 . We first sketch some features of the effects of initial coherences and dephasing rate on the purity decay (decoherence process), and then present the results of numerical calculations.

Noting that for a two-level system purity is given by $\varsigma=\frac{1}{2}\left(X^{2}+Y^{2}+Z^{2}+1\right)$ in the Bloch-vector representation and using Eqs. (6)-(8), one can obtain

$$
\frac{d \varsigma}{d t}=-4 \gamma\left(X^{2}+Y^{2}\right)
$$

From Eq.(17) one can expect that at fixed $\gamma$, for short times, purity decay gets faster with increasing $X_{0}$ and/or $Y_{0}$ under the restriction $X_{0}^{2}+Y_{0}^{2}+Z_{0}^{2} \leq 1$ (the equality holds only for the pure state). However, for the long time region the effects of $X_{0}$ and $Y_{0}$ on the purity decay are different due to different time-evolutions of $X$ and $Y$ (see Eqs.(9)-(11) 
and Eqs.(12)-(14)). While $X$ exponentially decays to zero (see Eq. (6)), $Y$ 's behavior over time is more complicated due to its coupling to $Z$ as seen in Eqs.(7) and (8). It is notable that purity decays invariantly with regard to $X_{0} \rightarrow-X_{0}$ regardless of time and dephasing rates, but not with regard to $Y_{0} \rightarrow-Y_{0}$. On the other hand, from Eq.(17) at a given initial condition purity is expected to decay faster with increasing dephasing rates $\gamma$ for short times such that $X \approx X_{0}$ and $Y \approx Y_{0}$. However, this tendency may change in the long time region, depending on conditions. Details of the effects of initial coherences, $\left(X_{0}, Y_{0}\right)$ and dephasing on the purity decay are explored below.

All numerical results are for initially pure states. One could also examine purity decay in initially mixed states but the initially pure state maximizes the influence of initial coherences and is inherently more interesting in terms of applications. We consider sets of initially pure states differing in the degree of $\mathrm{R}$ and $\mathrm{L}$ admixture. That is, $\varsigma(t=0)=1$ and thus $X_{0}^{2}+Y_{0}^{2}+Z_{0}^{2}=1$. Here $Z_{0}$ is chosen as $1-\sqrt{X_{0}^{2}+Y_{0}^{2}}$. Also for all the cases examined $\omega$ is chosen as 1 , and all the variables are in dimensionless units.

Figures 2 and 3 show purity as a function of initial coherences $\left(X_{0}, Y_{0}\right)$ with various dephasing rates spanning the tunneling dominant region $(\gamma<1$, (a) and (b)) to the dephasing dominant region $(\gamma>1,(\mathrm{c})$ and $(\mathrm{d}))$ at times $t=0.1$, and 1 , respectively. Several interesting observations are in order. First, one can easily see that for a given time and dephasing rates, purity is symmetric about $X_{0}=0$ at a fixed $Y_{0}$, but not symmetric about $Y_{0}=0$ at a fixed $X_{0}$. This implies that as $\left|X_{0}\right|$ increases at a fixed $Y_{0}=0$, purity decays faster for a given time and dephasing rate, but, however, as $\left|Y_{0}\right|$ increases at a fixed $X_{0}=0$, decay of purity may be suppressed. Figs. 3 (b) and (c) capture this feature very well. For instance, in Fig.3 (c) one can easily see that, as $Y_{0}$ increases in a negative direction from $Y_{0}=0$ with a fixed $X_{0}=0$, purity increases up to some point (i.e., purity increases with increasing $\left|Y_{0}\right|$ ), and then decreases. Second, for very short times such as $t=0.1$ [Fig. 2], purity tends to decay faster, as $\gamma$ increases and as $X_{0}^{2}+Y_{0}^{2}$ increases. This agrees well with the prediction of Eq. (17), which is $\varsigma(t) \approx 1-4 \gamma\left(X_{0}^{2}+Y_{0}^{2}\right) t$ for very short times such that $X \approx X_{0}$ and $Y \approx Y_{0}$. Also note that this proportionality to $X_{0}^{2}+Y_{0}^{2}$ gets distorted for the larger $\gamma$; the circle in the contour of the purity gets distorted in Figs. 2 (c), (e) and (d), (f). This is because increasing $\gamma$ accelerates changes of $X, Y$ from $X_{0}, Y_{0}$. Third, on the other hand, for later times (see Fig. 3), and in the region of larger initial coherences $\left(X_{0}^{2}+Y_{0}^{2} \approx 1\right)$, purity still tends to decay faster with increasing $\gamma$, but for the smaller initial 
coherences $\left(X_{0}^{2}+Y_{0}^{2} \approx 0\right)$, purity shows the opposite behavior, with increasing $\gamma$ beyond $\omega$, i.e. , the purity decay starts to be suppressed. That is, if initially the system is strongly localized in one of the two wells (smaller initial coherences), then introducing sufficiently large dephasing (considerably larger than tunneling frequency) will suppress the decoherence process (racemization), but, however, if initially the system is strongly delocalized in two wells (larger initial coherences), then increasing the dephasing rate could accelerate the decoherence process and the purity decay at a rate faster than for the initially well-localized system for a given condition. This different behavior of the system in respect to increasing $\gamma$, depending on its initial condition, can be understood by recognizing the role of the environment as destroying the left-right coherences, and thus inhibiting tunneling of the system. The tunneling (transition) suppression leads to the stabilization of the chirality for the initially localized state (or so-called quantum Zeno effect) and the faster racemization for the initially delocalized state. Implications of this interesting result contribute to answers to some of long-standing fundamental problems in chiral systems: 1) why are the chiral states of some molecules, once produced, stable for a very long time? 2) why are superpositions of stable chiral states not observed? Our result suggests that the system, if it is initially a welllocalized state, will tend to preserve the chirality in the external environment that destroys the left-right coherences, provided that the interaction with the environment dominates the tunneling interaction, while, on the other hand, the system, if initially a superposition of two chiral states, would become very quickly a racemic mixture in the presence of the very same external environment.

\section{SUMMARY}

We have obtained an analytical solution for an open chiral system. The interaction of the system with an environment was modeled as a continuous position measurement of the system and a two-level approximation was made for the system. The interplay of the initial coherences and the decoherence on the distinguishability between the pure/mixed states, and the racemization/stabilization of the chirality was clarified. If the two chiral states are initially taken to be real for the pure state (i.e. , $Y_{0}^{\mathrm{P}}=0$ ), no distinguishability was shown to exist for all time and the dephasing rate, as measured by a population difference between the two chiral states, was essentially zero. Increasing the dephasing rate was shown 
to tend to accelerate the distinguishability decay for $Y_{0}^{\mathrm{P}} \neq 0$, and the purity decay over time. However, considerably strong dephasing (beyond a certain threshold) was observed to enable the long-time survival of distinguishability, and the chirality stabilization for the initially localized state. The results should serve as a prototype for understanding the results of a chiral system under more complicated and realistic conditions, e.g. , more energy levels, more spatial degrees of freedom, and other types of external environment which give rise to chiral or achiral interactions.

This work may be extended to a chiral system interacting with lasers. Recently there has been considerable interest on the preparation/control of molecular chirality [6-9]. However,

the decoherence effect on these preparation/control scenarios is far from well understood, especially when the time scales of system dynamics, and/or environmental effects (energy loss, dephasing) are comparable to the interaction time scale between the system and the laser field.

\section{Acknowledgements}

It is a pleasure to acknowledge the Natural Sciences and Engineering Research Council of Canada for financial support in the form of a Discovery Grant. Authors thank Alexei M. Frolov (UWO) for consultations on aspects of this manuscript. 
[1] J.J. Sakurai, Modern Quantum Mechanics (Addison-Wesley Publishing Company, Inc., 1985), Chap 4.

[2] F. Hund, Z. Phys. 43, 805 (1927).

[3] R. Silbey and R. A. Harris, J. Phys. Chem. 93, 7062 (1989).

[4] P. Pfeifer, Phys. Rev. A. 26,701 (1982).

[5] B. Fain, Phys. Lett. A 89, 455 (1982).

[6] J. A. Cina and R. A. Harris, J. Chem. Phys. 100, 2531 (1994).

[7] R. P. Duarte-Zamorano and V. V. Romero-Rochin, J. Chem. Phys. 114, 9276 (2001).

[8] M. Shapiro, E. Frishman, and P. Brumer, Phys. Rev. Lett. 84, 1669 (2000).

[9] Y. Fujimura, L. Gonalez, K. Hoki, J. Manz, and Y. Ohtsuki, Chem. Phys. Lett. 306, 1 (1999).

[10] I.O. Stamatescu, E. Joos, H.D. Zeh, C. Kiefer, D. Giulini, and J. Kupsch, Decoherence and the Appearance of a Classical World in Quantum Theory, 2nd ed. (Springer Verlag, 2003).

[11] H. Han and P. Brumer, J. Chem. Phys. 122144316 (2005) and references are therein.

[12] M. J. Gagen, H. M. Wiseman, and G. J. Milburn, Phys. Rev. A 48, 132 (1993).

[13] G. J. Milburn, J. Opt. Soc. Am. B 5, 1317 (1988).

[14] E. Joos and H.D. Zeh, Z. Phys. B 59, 223 (1985).

[15] A.O. Caldeira and A.J. Leggett, Physica A 121.

[16] W. H. Zurek, Rev. Mod. Phys. 75, 715 (2003) and references are therein.

[17] M. R. Gallis and G. N. Fleming, Phys. Rev. A 42, 38 (1990).

[18] M. O. Scully and M. S. Zubairy, Quantum optics (Cambridge University Press, 1997).

[19] L. Allen and J. H. Eberly, Optical Resonance and Two-Level Atoms (Wiley, New York, 1975).

[20] H. C. Torrey, Phys. Rev. 76, 1059 (1949).

[21] R. A. Harris, Y. Shi, and J. A. Cina, J. Chem. Phys. 101, 3459 (1994).

[22] W.H. Zurek, S. Habib and J.P. Paz, Phys. Rev. Lett. 70, 1187 (1993). 

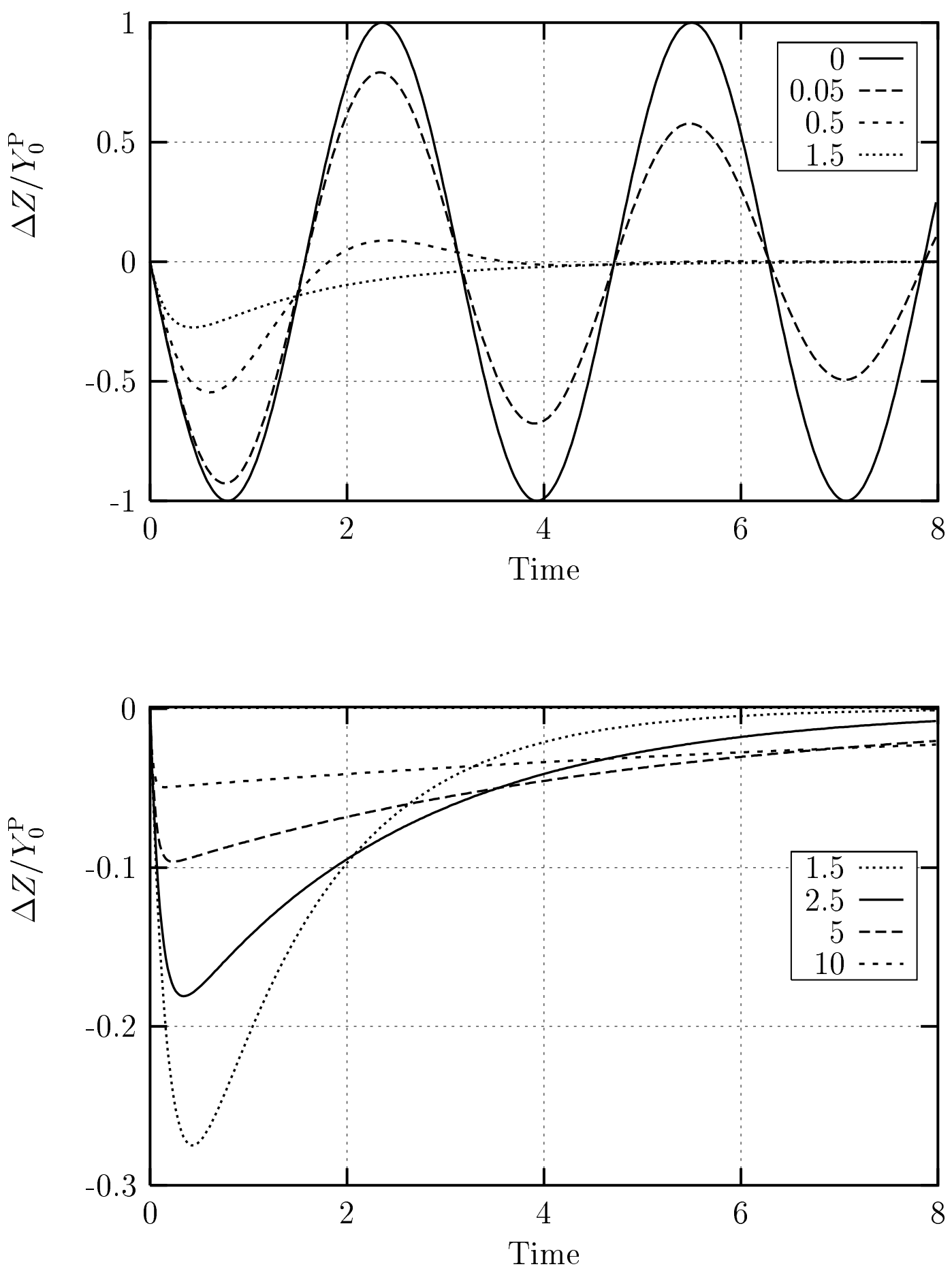

FIG. 1: $\Delta Z(t) / Y_{0}^{\mathrm{P}}$ vs. time for various $\gamma$ shown inside the box. Upper panel: $\gamma=0$ (dephasingfree case), $\gamma=0.05,0.5$ (tunneling dominant region), and $\gamma=1.5$ (dephasing dominant region). Lower panel: all are in dephasing dominant region. All the variables are in dimensionless units. 
(a) $\gamma=0.025$

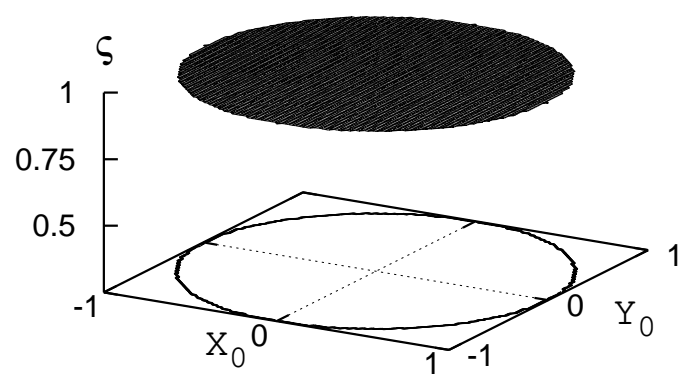

(c) $\gamma=1.25$

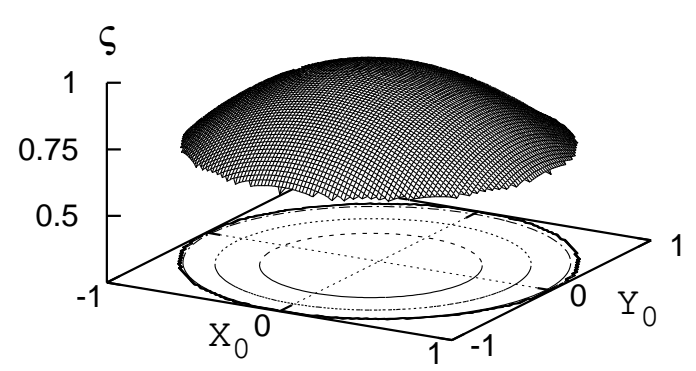

(e) $\gamma=1.25$

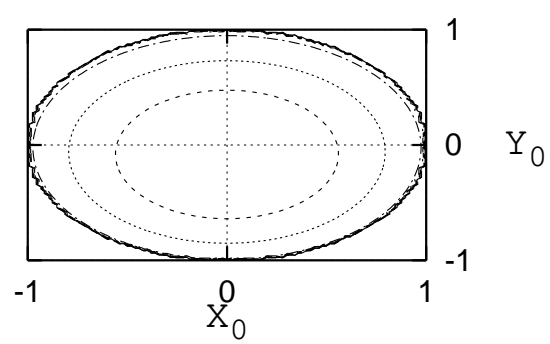

(b) $\gamma=0.25$

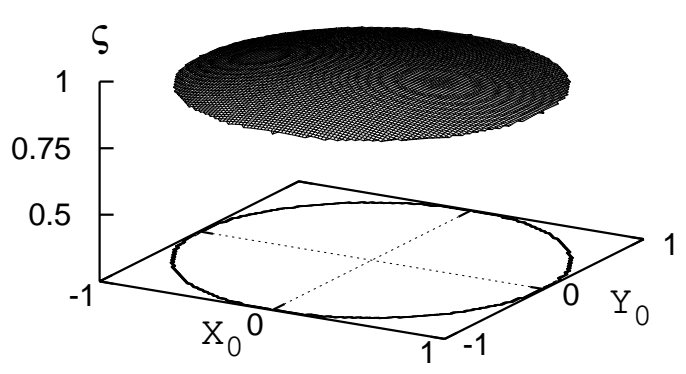

(d) $\gamma=2.5$

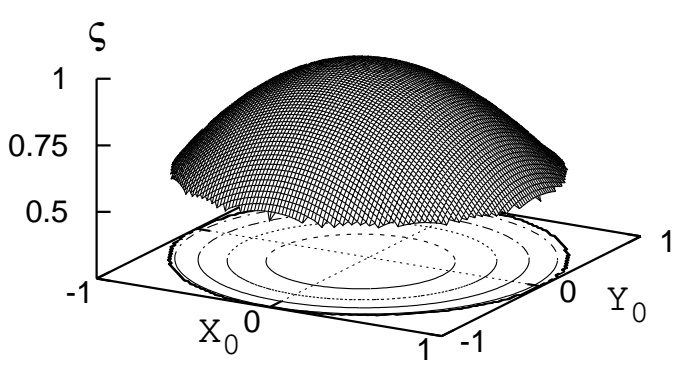

(f) $\gamma=2.5$

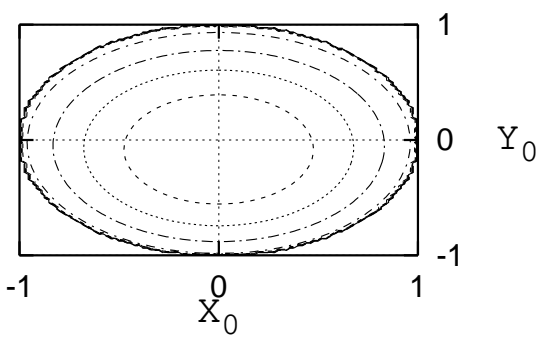

FIG. 2: Purity $\varsigma$ vs. initial coherences $\left(X_{0}, Y_{0}\right)$ at time $t=0.1$ for various dephasing rates $\gamma$ : (a) $\gamma=0.025$, (b) $\gamma=0.25$, (c) $\gamma=1.25$, and (d) $\gamma=2.5$ ((e) and (f) are 2-dimensional contour plots for (c) and (d), respectively). All the variables are in dimensionless units. 
(a) $\gamma=0.025$

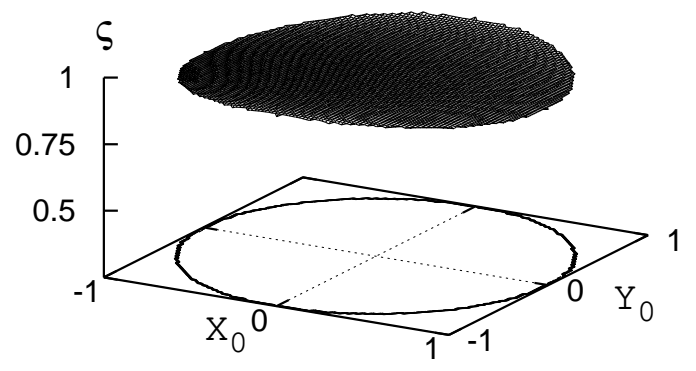

(c) $\gamma=1.25$

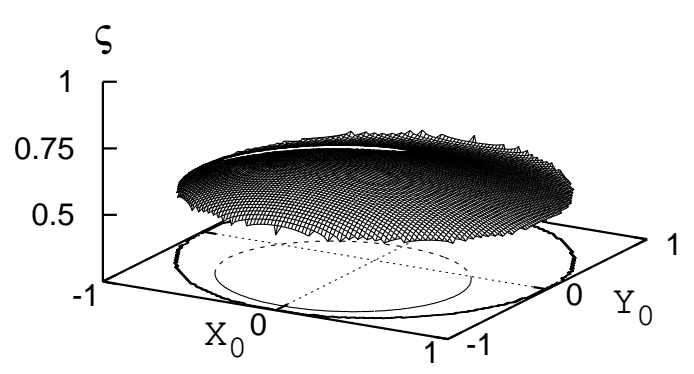

(b) $\gamma=0.25$

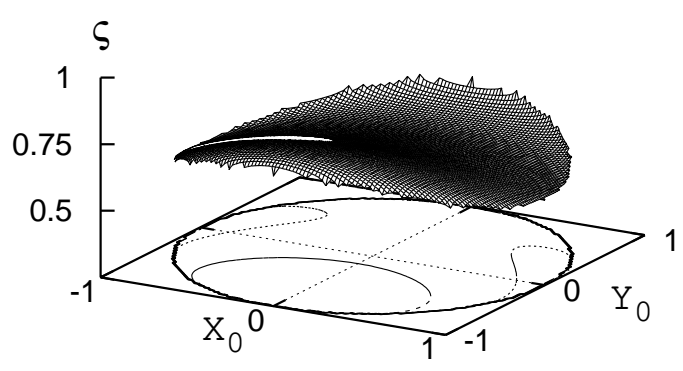

(d) $\gamma=2.5$

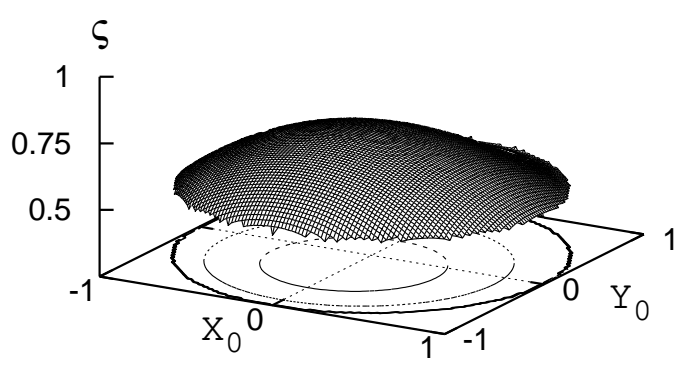

FIG. 3: As in Fig. 2 but at time $t=1$. 
(f) $\gamma=2.5$

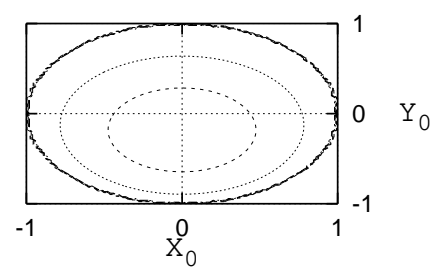


(e) $\gamma=1.25$

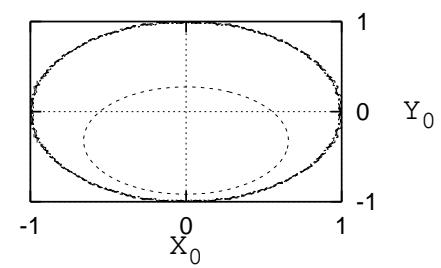

